\title{
Nonsmooth optimal value and policy functions in mechanical systems subject to unilateral constraints
}

\author{
Bora S. Banjanin
}

\begin{abstract}
State-of-the-art approaches to optimal control use smooth approximations of value and policy functions and gradient-based algorithms for improving approximator parameters. Unfortunately, we show that value and policy functions that arise in optimal control of mechanical systems subject to unilateral constraints - i.e. the contact-rich dynamics of robot locomotion and manipulation - are generally nonsmooth due to the underlying dynamics exhibiting discontinuous or piecewisedifferentiable trajectory outcomes. Simple mechanical systems are used to illustrate this result and the implications for optimal control of contact-rich robot dynamics.
\end{abstract}

\section{INTRODUCTION}

This paper focuses on optimal control of mechanical systems subject to unilateral constraints [1], which are commonly used to model contact-rich dynamics of rigid robots [2]. In an optimal control problem, a policy is sought that extremizes a given performance criterion; the performance achieved by this optimal policy is the optimal value of the problem. Two popular approaches for solving such problems are trajectory optimization [3] and reinforcement learning [4]. Although many algorithms are available in either framework, scalable algorithms in both leverage local approximations - gradients of values and/or policies - to iteratively improve toward optimality. In applications with smooth dynamics, these gradients are guaranteed to exist and can be readily computed or approximated.

Recent work has applied state-of-the-art algorithms for trajectory optimization [5]-[8] and reinforcement learning [9][12] to optimal control of contach-rich dynamics, producing impressive results in simulations and experiments of robot manipulation and locomotion. However, the algorithms underlying these results [3], [13] are only known to converge to stationary points in smooth systems since they rely on gradients of the functions that define costs and constraints.

\section{A. Our contributions}

We show that these gradients generally fail to exist for mechanical systems subject to unilateral constraints due to nonsmoothness in the underlying dynamics; this result is derived theoretically in Theorem 1 and demonstrated using the simple mechanical system depicted in Fig. 11. These contributions imply that additional work is required to justify applying state-of-the-art algorithms for optimal control to mechanical systems with contact-rich robot dynamics.

Dept. of Electrical \& Computer Engineering, University of Washington, Seattle, WA, USA (borab, sburden@uw . edu). +1 (206) 225 3545. This material is based upon work supported by the U. S. Army Research Office under grant number W911NF-16-1-0158.

\author{
Samuel A. Burden
}

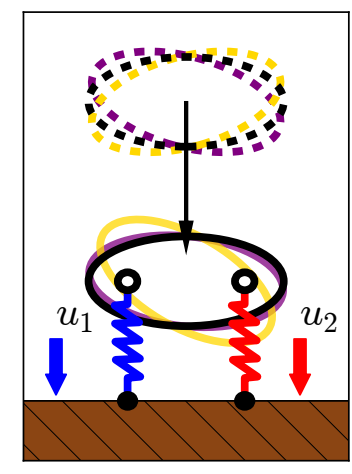

(a) touchdown maneuver

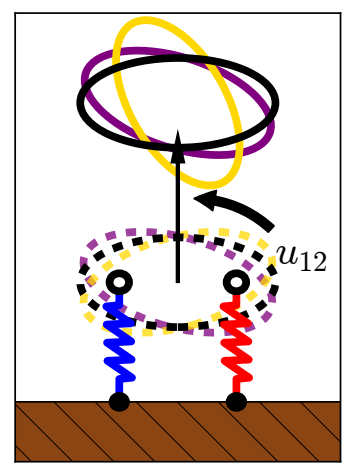

(b) liftoff maneuver
Fig. 1. Saggital-plane biped performs two maneuvers with contactrich dynamics - (a) touchdown and (b) liftoff - using policies that exert different forces depending on which feet are in contact with the ground. In the touchdown maneuver, feet are initially off the ground and trajectories terminate when the body height reaches nadir; in the liftoff maneuver, feet are initially on the ground and trajectories terminate when the body height reaches apex. Fig. 2 shows that the final body rotation is a nonsmooth piecewise-differentiable or discontinuous - function of initial body rotation.

\section{B. Organization}

We begin in Sec. II by modeling contact-rich robot dynamics using mechanical systems subject to unilateral constraints, and describe how nonsmoothness - discontintinuity or piecewise-differentiability - manifests in trajectory outcomes and (hence) trajectory costs. Then in Sec. III we provide mathematical derivations that show nonsmoothness in trajectory outcomes and costs gives rise to nonsmoothness in optimal value and (hence) policy functions. Subsequently in Sec. IV] we present numerical simulations that demonstrate discontinuous or merely piecewise-differentiable optimal value and policy functions in a mechanical system subject to unilateral constraints. Finally in Sec. $\mathrm{V}$ we discuss the prevalence of nonsmoothness in applications and how the lack of classical differentiability prevents gradient-based algorithms from converging to optimality.

\section{CONTACT-RICH ROBOT DYNAMICS}

In this section, we formalize a class of models for contactrich dynamics in robot locomotion and manipulation as mechanical systems subject to unilateral constraints and formulate an optimal control problem for these systems.

\section{A. Dynamics}

Consider the dynamics of a mechanical system with $d \in \mathbb{N}$ degrees-of-freedom (DOF) $q \in Q=\mathbb{R}^{d}$ subject to $n \in \mathbb{N}$ 


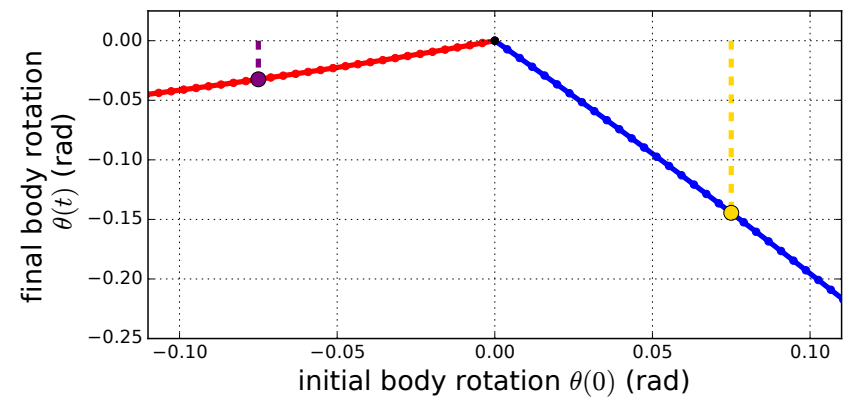

(a) touchdown trajectory outcomes

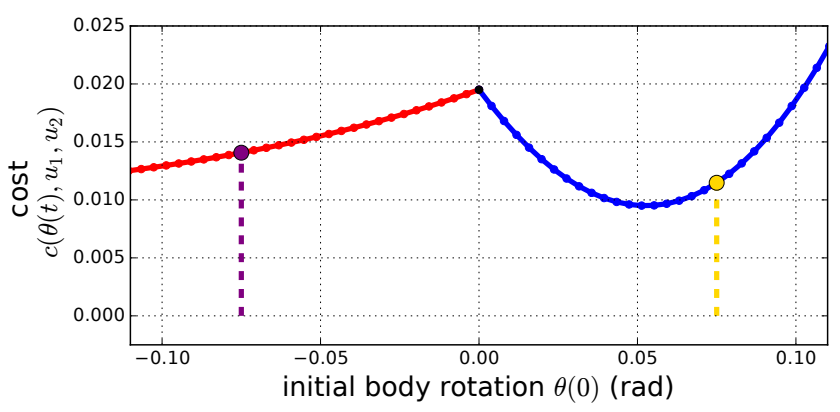

(c) touchdown value

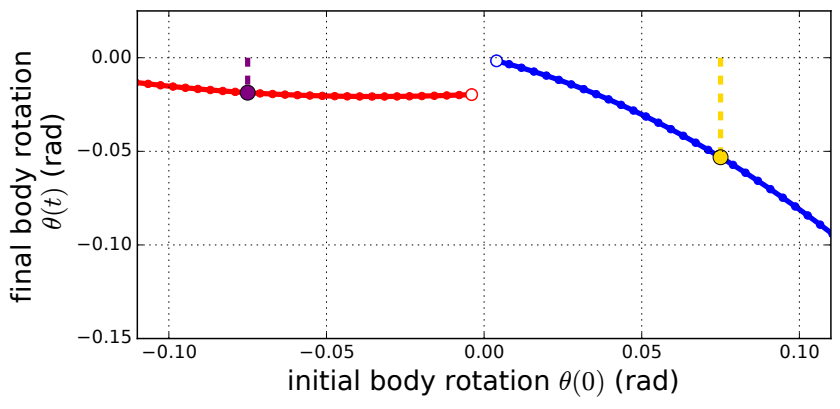

(b) liftoff trajectory outcomes

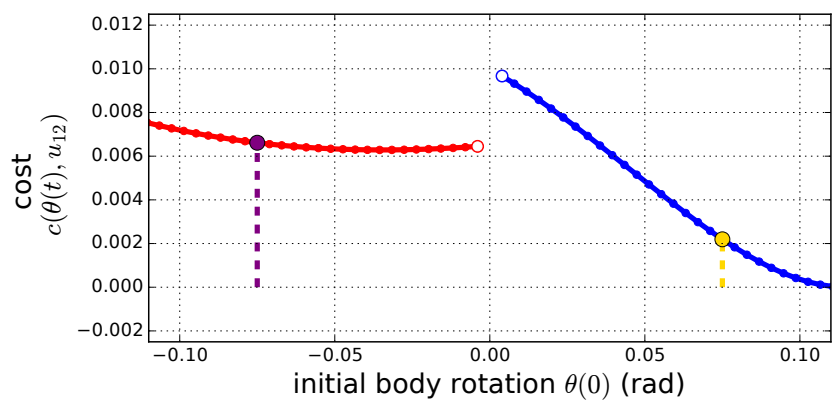

(d) liftoff value

Fig. 2. Piecewise-differentiable and discontinuous trajectory outcomes in the saggital-plane biped from Fig. 1, (a,b) Trajectory outcomes (final body angle $\theta(t)$ ) as a function of initial body angle $\theta(0)$. (c,d) Performance of trajectories as measured by the cost functions in 14, , 15. Dashed colored vertical lines indicate corresponding colored outcomes in Fig. 1 and dashed lines in Fig. 3

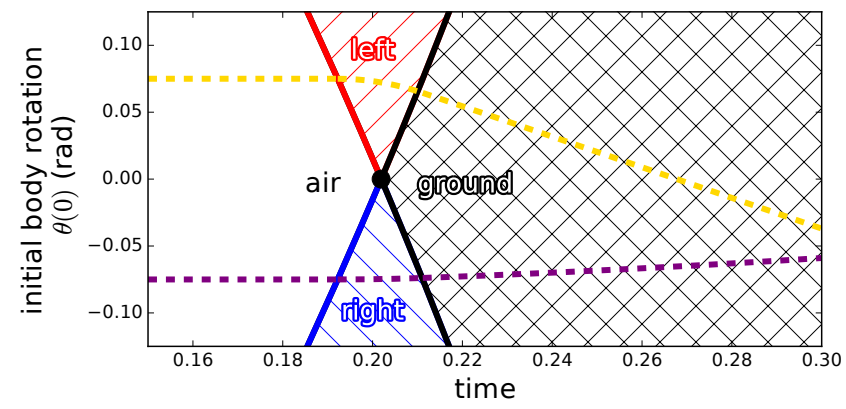

(a) touchdown contact modes

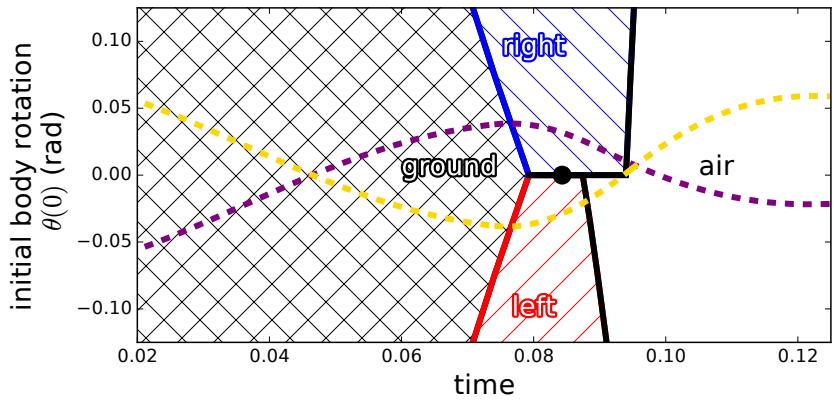

(b) liftoff contact modes

Fig. 3. Contact modes for touchdown and liftoff maneuvers. The saggital-plane biped illustrated in Fig. 11a,b) can be in one of four contact modes corresponding to which subset $J \subset\{1,2\}$ of the (two) limbs are in contact with the ground; each subset yields different dynamics in 11 . (a,b) System contact mode at each time $t$ for a given initial body rotation $\theta(0)$; the body torque input is zero $\left(u_{12}=0\right)$ and the leg forces are different $\left(u_{1} \neq u_{2}\right)$ in mode left $(\{1\})$ and right $(\{2\})$ than in air $(\emptyset)$ or ground $(\{1,2\})$. Dashed colored horizontal lines indicate corresponding colored trajectories in Fig. 1 The increase in force during the transition to modes left and right in (b) changes the ground reaction force discontinuously, delaying liftoff and causing discontinuous trajectory outcomes in Fig. 11d).

unilateral constraints $a(q) \geq 0$ specified by a continuouslydifferentiable function $a: Q \rightarrow \mathbb{R}^{n}$, where the inequality is enforced componentwise. Given any $J \subset\{1, \ldots, n\}$, and letting $|J|$ denote the number of elements in the set $J$, we let $a_{J}: Q \rightarrow \mathbb{R}^{|J|}$ denote the function obtained by selecting the component functions of $a$ indexed by $J$. It is well-known (cf. [1, Sec. 3] or [2, Sec. 2.4, 2.5]) that, with $J=\left\{j \in\{1, \ldots, n\}: a_{j}(q)=0\right\}$ denoting the contact mode, the system's dynamics take the form

$$
\begin{aligned}
M(q) \ddot{q} & =f_{J}(q, \dot{q}, u)+D a_{J}(q)^{\top} \lambda_{J}(q, \dot{q}, u), \\
\dot{q}^{+} & =\Delta_{J}(q) \dot{q}^{-},
\end{aligned}
$$

where: $M(q) \in \mathbb{R}^{d \times d}$ is the mass matrix; $f_{J}(q, \dot{q}, u) \in \mathbb{R}^{d}$ is the vector of Coriolis, potential, and applied forces; $u \in \mathcal{U}$ is an external input, $D a_{J}(q) \in \mathbb{R}^{|J| \times d}$ denotes the derivative of the constraint function $a_{J} ; \lambda_{J}(q, \dot{q}, u) \in \mathbb{R}^{|J|}$ denotes the reaction forces generated in contact mode $J$ to enforce $a_{J}(q) \geq 0 ; \Delta_{J}(q) \in \mathbb{R}^{d \times d}$ specifies the collision restitution law that instantaneously resets velocities to ensure compat- 
ibility with the constraint $a_{J}(q)=0$; and $\dot{q}^{+}$(resp. $\dot{q}^{-}$) denotes the right- (resp. left-)handed limits of the velocity with respect to time. Note that we explicitly allow the dynamics in (1) to vary with contact mode $J \subset\{1, \ldots, n\}$.

\section{B. Properties of dynamics}

The seemingly benign equations in (1) can yield dynamics with a range of regularity properties. This issue has been investigated elsewhere [1], [14], [15]; here we focus specifically on how the design of a robot's mechanical and control systems affect (non)smoothness of trajectory outcomes.

It is common to assume that the functions in (1) are continuously-differentiable; however, as illustrated by [1, Ex. 2], this assumption alone does not ensure even existence or uniqueness of trajectories, let alone smoothness of trajectory outcomes. This case contrasts starkly with that of a smooth differential or difference equation

$$
\dot{x} \text { or } x^{+}=F(x, u),
$$

which yields unique trajectories that vary smoothly with respect to state $x \in \mathcal{X}$ and control input $u \in \mathcal{U}$ [3, Thm. 5.6.8]. Since we are chiefly concerned with how properties of the dynamics in (1) affect properties of optimal value and policy functions, we will assume ${ }^{1}$ in what follows that conditions have been imposed to ensure unique trajectories of (1) exist for time horizons, initial states, and control inputs of interest.

Assuming that unique trajectories exist for (1) does not provide any regularity properties on the trajectory outcomes; these properties are determined by the design of a robot's mechanical and control systems and their closed-loop interaction with the environment. For instance: when limbs are inertially coupled (e.g. by rigid struts and joints), so that one limb's constraint activation instantaneously changes another's velocity, trajectories can vary discontinuously near configurations where these two limbs activate constraints simultaneously [16, Table 3] [17]; when limbs are force coupled (e.g. by a mode-switching controller), so that one limb's constraint (de)activation instantaneously changes the force on another, trajectories can vary piecewise-differentiably near configurations where these two limbs (de)activate constraints simultaneously [14, Fig. 1]. It is this force coupling, explicitly permitted by the mode-dependence of the dynamics in (1), that we will leverage to obtain nonsmooth trajectory outcomes in the examples presented in Sec. IV

\section{Properties of optimal value and policy functions}

A broad class of optimal control problems for the dynamics in (1) can be formulated in terms of final $(\ell: X \rightarrow \mathbb{R})$ and running $(\mathcal{L}:[0, t] \times \mathcal{X} \times \mathcal{U} \rightarrow \mathbb{R})$ costs:

$$
\nu(x)=\min _{u \in \mathcal{U}[0, t]} \ell\left(\phi^{x, u}(t)\right)+\int_{0}^{t} \mathcal{L}\left(s, \phi^{x, u}(s), u\right) d s,
$$

where $\phi^{x, u}:[0, t] \rightarrow X$ denotes the unique trajectory obtained from initial state $\phi^{x, u}(0)=x \in \mathcal{X}$ when input $u \in \mathcal{U}$ is applied. To expose the dependence of the cost

\footnotetext{
${ }^{1}$ We refer the interested reader to $[1, \mathrm{Thm} .10]$ or [2] for conditions that ensure existence and uniqueness of trajectories of [1].
}

in (3) on the trajectory outcome function $\phi$, we transcribe the problem in (3) to a simpler form using a standard state augmentation technique [3, Ch. 4.1.2],

$$
\nu(x)=\min _{u \in \mathcal{U}} c(\phi(t, x, u)),
$$

where $\phi:[0, t] \times \mathcal{X} \times \mathcal{U} \rightarrow \mathcal{X}$ is the flow function defined by $\phi(t, x, u)=\phi^{x, u}(t)$. As discussed in Sec. II-B, the properties of $\phi$ are determined by a robot's design: it is possible for $\phi$ and hence $c \circ \phi$ to be discontinuous $\left(\phi \notin C^{0}\right)$ or piecewise-differentiable and not continuously-differentiable $\left(\phi \in P C^{r} \backslash C^{r}\right.$ ) depending on the properties of the robot's mechanical and control systems. In the next section, we study how continuity and differentiability properties of the cost $c \circ \phi$ affect the corresponding properties of the value $\nu$ in (4).

\section{NONSMOOTH OPTIMAL VALUE \& POLICY FUNCTIONS}

Consider minimization of the cost function $c: \mathcal{X} \times \mathcal{U} \rightarrow \mathbb{R}$ with respect to an input $u \in \mathcal{U}$ :

$$
\nu(x)=\min _{u \in \mathcal{U}} c(x, u)
$$

so long as $\mathcal{X}$ and $\mathcal{U}$ are compact and $c$ is continuous, the function $\nu: X \rightarrow \mathbb{R}$ indicated in (5), termed the optimal value function, is well-defined. We let $\pi: \mathcal{X} \rightarrow \mathcal{U}$ denote an optimal policy for (5), i.e.

$$
\forall x \in X: \pi(x) \in \arg \min _{u \in \mathcal{U}} c(x, u)
$$

or, equivalently,

$$
\forall x \in \mathcal{X}: \nu(x)=c(x, \pi(x)) .
$$

In this section we study how continuity and differentiability properties of the cost function $(c)$ relate to corresponding properties of the optimal value $(\nu)$ and policy $(\pi)$ functions.

\section{A. Discontinuous cost functions}

If the cost $(c: \mathcal{X} \times \mathcal{U} \rightarrow \mathbb{R})$ is discontinuous with respect to its first argument, then the optimal policy $(\pi: \mathcal{X} \rightarrow \mathcal{U})$ and value $(\nu: X \rightarrow \mathbb{R})$ are generally discontinuous as well. This observation is clear in the trivial case that the cost only depends on its first argument, but manifests more generally.

\section{B. Piecewise-differentiable cost functions}

If $c$ is piecewise-differentiable ${ }^{2}$ which we denote by $c \in P C^{1}(X \times \mathcal{U}, \mathbb{R})$ or simply $c \in P C^{1}$, then necessarily

$$
\forall w \in T_{u} \mathcal{U}: D_{2} c(x, \pi(x) ; w) \geq 0 .
$$

Here, $D_{2} c(x, \pi(x)): T_{u} \mathcal{U} \rightarrow \mathbb{R}$ denotes a continuous and piecewise-linear first-order approximation termed the Bouligand (or $B$-)derivative [18, Ch. 3] that exists by virtue of the cost being $P C^{1}$ [18, Lem. 4.1.3]; $D_{2} c(x, \pi(x) ; w)$ denotes the evaluation of $D_{2} c(x, \pi(x))$ at $w \in T_{u} \mathcal{U}$.

\footnotetext{
${ }^{2}$ We use the notion of piecewise-differentiability from [18, Ch. 4.1]: a function is piecewise-differentiable if it is everywhere locally a continuous selection of a finite number of continuously-differentiable functions.
} 
If $c$ is two times piecewise-differentiable $\left(c \in P C^{2}\right.$ ), and if a sufficient condition [19, Thm. 1] for strict local optimality for (5) is satisfied at $\pi(x) \in \mathcal{U}$,

$$
\begin{array}{r}
\forall w \in\left\{w \in T_{u} \mathcal{U} \mid w \neq 0, D_{2} c(x, \pi(x) ; w)=0\right\} \\
: D_{2}^{2} c(x, \pi(x) ; w, w)>0,
\end{array}
$$

and if the piecewise-linear function

$$
D_{2}^{2} c(x, \pi(x)): T_{u} \mathcal{U} \rightarrow T_{u} \mathcal{U} \text { is invertible, }
$$

then a $P C^{1}$ Implicit Function Theorem can be applied to choose $\pi \in P C^{1}$ near $x$ [20, Cor. 3.4]. Applying the $P C^{1}$ Chain Rule [18, Thm. 3.1.1] to (8) yields (cf. [20, § 3])

$$
\begin{aligned}
& \forall v \in T_{x} X: D \pi(x ; v)= \\
& \quad-D_{2}^{2} c(x, \pi(x))^{-1}\left(D_{12} c(x, \pi(x) ; v)\right),
\end{aligned}
$$

and applying the $P C^{1}$ Chain Rule to (7) yields

$$
\begin{aligned}
& \forall v \in T_{x} X: D \nu(x ; v)=D_{x} c(x, \pi(x) ; v) \\
& \quad=D_{1} c(x, \pi(x) ; v)+D_{2} c(x, \pi(x) ; D \pi(x ; v)),
\end{aligned}
$$

whence we obtain B-derivatives of the optimal value and policy functions in terms of B-derivatives of the cost.

We conclude that if the cost function is two times piecewise-differentiable $\left(c \in P C^{2}\right)$ and first-order necessary (8) and second-order sufficient $(9),(10)$ conditions for optimality and stability of solutions to (5) are satisfied at $u=\pi(x)$, then the optimal policy and value functions are piecewise-differentiable at $x\left(\pi, \nu \in P C^{1}\right)$ and their Bderivatives at $x$ can be computed using (11), (12).

Theorem 1: If $c \in P C^{2}(X \times \mathcal{U}, \mathbb{R})$ satisfies (8), (9), and $(10)$ at $(\xi, \mu) \in \mathcal{X} \times \mathcal{U}$, then there exist neighborhoods $X \subset \bar{X}$ of $\xi$ and $U \subset \mathcal{U}$ of $\mu$ and a function $\pi \in P C^{1}(X, U)$ such that $\pi(\xi)=\mu$ and, for all $x \in X, \pi(x)$ is the unique minimizer for

$$
\nu(x)=\min _{u \in U} c(x, u)
$$

the B-derivative of $\pi$ is given by (11), and the B-derivative of $\nu$ is given by (12).

\section{Conclusions about optimal value \& policy functions}

The results in Sections III-A and III-B suggest that we should generally expect continuity and differentiability properties of optimal value and policy functions to match that of the cost function: they should be discontinuous when the cost is discontinuous, or piecewise-differentiable when the cost is piecewise-differentiable. In Sec. IV] we demonstrate these effects in the class of models described in Sec. III.

\section{NONSMOOTH OPTIMAL VALUE \& POLICY FUNCTIONS IN CONTACT-RICH ROBOT DYNAMICS}

We showed in the previous two sections that optimal value and policy functions inherit nonsmoothness from the underlying dynamics. To instantiate this result, we crafted a simple mechanical system subject to unilateral constraints that exhibits piecewise-differentiable and discontinuous trajectory outcomes, yielding the touchdown and liftoff maneuvers shown in Fig. 11(a,b). For the touchdown maneuver, we seek the optimal (constant) force to exert in the left leg $\left(u_{1}\right)$ when the left foot is in contact and the right foot is not; similarly, we seek the optimal choice of force in the right leg $\left(u_{2}\right)$ when the right foot is in contact and the left foot is not: with $\theta^{*}$ denoting the desired body rotation at nadir and $\alpha_{1}, \alpha_{2}>0$ denoting input penalty parameters,

$$
c_{\text {touchdown }}\left(\theta, u_{1}, u_{2}\right)=\left(\theta-\theta^{*}\right)^{2}+\alpha_{1} u_{1}^{2}+\alpha_{2} u_{2}^{2} .
$$

For the liftoff maneuver, we seek the optimal (constant) torque $\left(u_{12}\right)$ to apply to the body while both feet are in contact: with $\theta^{*}$ denoting the desired body rotation at apex and $\alpha_{12}>0$ denoting an input penalty parameter,

$$
c_{\text {liftoff }}\left(\theta, u_{12}\right)=\left(\theta-\theta^{*}\right)^{2}+\alpha_{12} u_{12}^{2} .
$$

We implemented numerical simulations of these model ${ }^{3}$ and applied a scalar minimization algorithm ${ }^{4}$ to compute optimal policies as a function of initial body rotation.

As expected, the optimal value and policy functions computed for the touchdown and liftoff maneuvers are nonsmooth (Fig. $4(\mathrm{c}, \mathrm{d}, \mathrm{e}, \mathrm{f})$ ), owing to the nonsmoothness of the optimal trajectory outcomes (Fig. 4 (a,b)). This result does not depend sensitively on the problem data; nonsmoothness is preserved after altering parameters of the model and/or cost functions. We emphasize that the nonsmoothness in Fig. 4 arises from the nonsmoothness in the underlying system dynamics (11), as the functions in (14) and (15) are smooth.

\section{DISCUSSION}

We conclude by discussing what our results imply about the use of smooth tools in nonsmooth settings (Sec. $\mathrm{V}-\mathrm{A}$ ) and how often we expect to encounter the nonsmooth phenomena described above in models of robot behaviors (Sec. V-B).

\section{A. Justifying the use of gradient-based algorithms}

Suppose a (possibly non-optimal) policy $\pi: \mathcal{X} \rightarrow \mathcal{U}$ has an associated value $\nu^{\pi}: \mathcal{X} \rightarrow \mathbb{R}$. If this value admits a firstorder approximation with respect to $\pi$, then it is natural to improve the policy by descending the cost landscape: with $\alpha>0$ as a stepsize parameter,

$$
\pi^{+}=\pi+\alpha \arg \min _{\|\delta\|=1} D_{\pi} \nu^{\pi}(\delta) .
$$

The update in 16 is a direct policy gradient-based algorithm [22], [23], and can be interpreted as a natural [24] or trust region [9] algorithm depending on the norm chosen. In practice, the derivative $D_{\pi} \nu^{\pi}$ is not known in closedform and must be estimated, e.g. using function approximation [25], [26] or sampling [23], [27]. This practice is justified for smooth control systems; it is not generally justified for the mechanical systems subject to unilateral constraints considered here since the value of (optimal or non-optimal) policies can be nonsmooth.

Recent work employs smooth approximations of the contact-rich robot dynamics in (11) to enable application of gradient-based learning [10], [11], [28] and optimization [7], [29], [30] algorithms. This approach leverages established

\footnotetext{
${ }^{3}$ using the modeling framework in [2] and simulation algorithm in [21]

${ }^{4}$ Scipy v0.19.0 minimize_scalar
} 


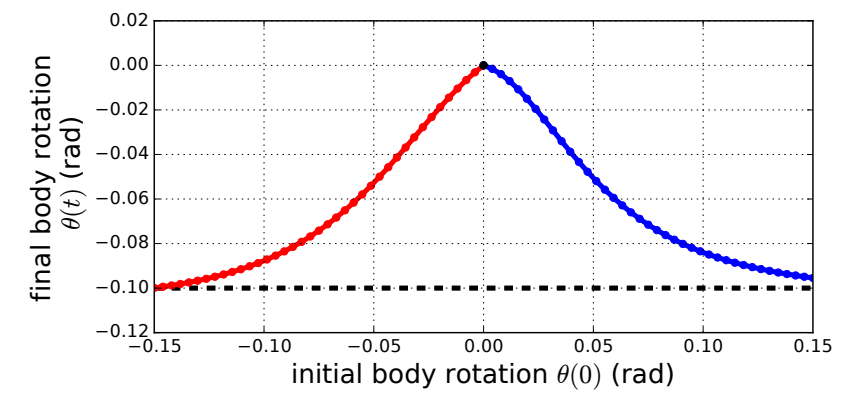

(a) optimal touchdown trajectory outcomes

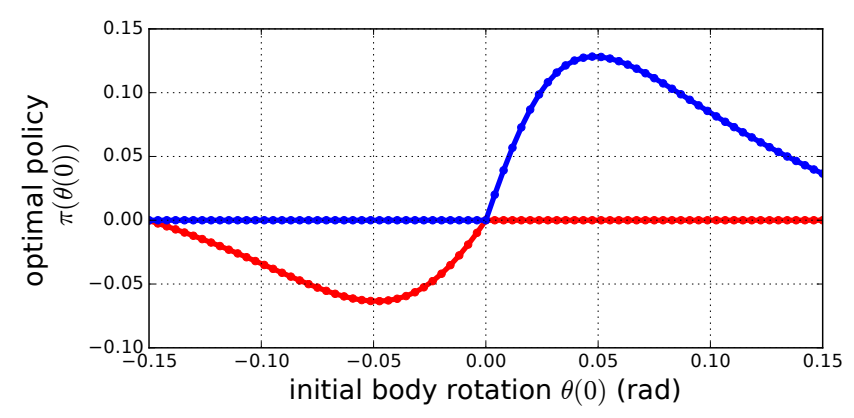

(c) optimal touchdown policy

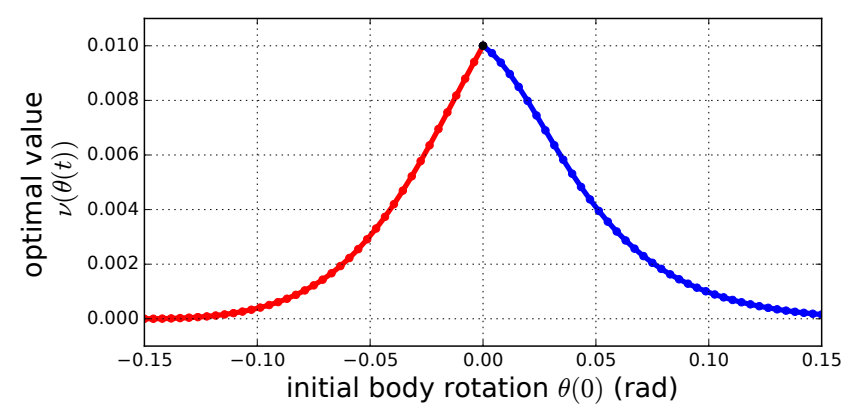

(e) optimal touchdown value

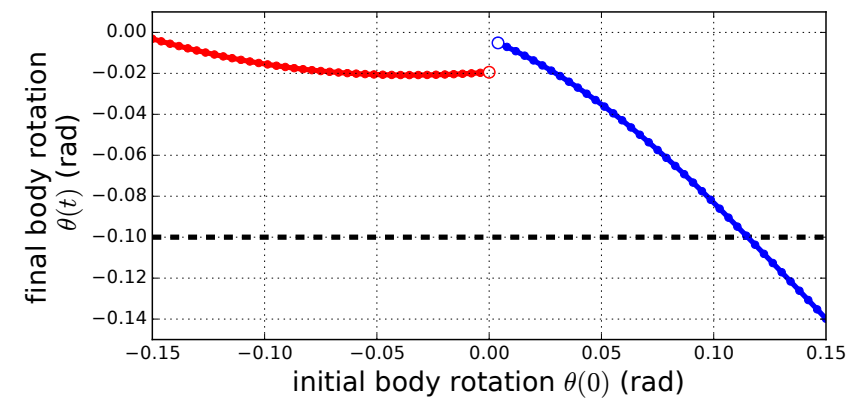

(b) optimal liftoff trajectory outcomes

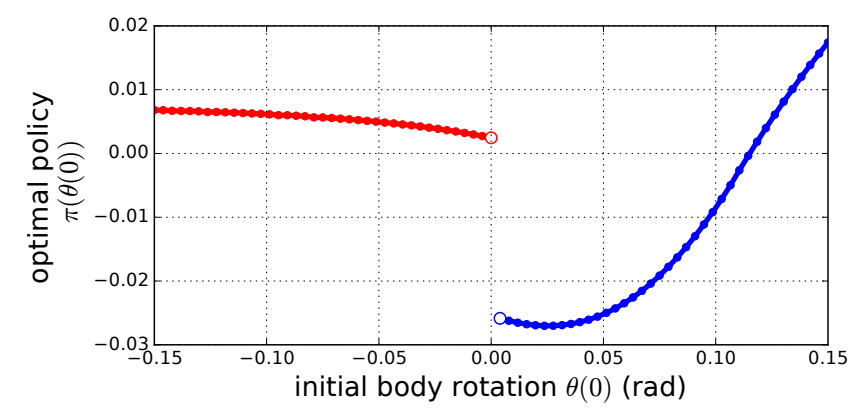

(d) optimal liftoff policy

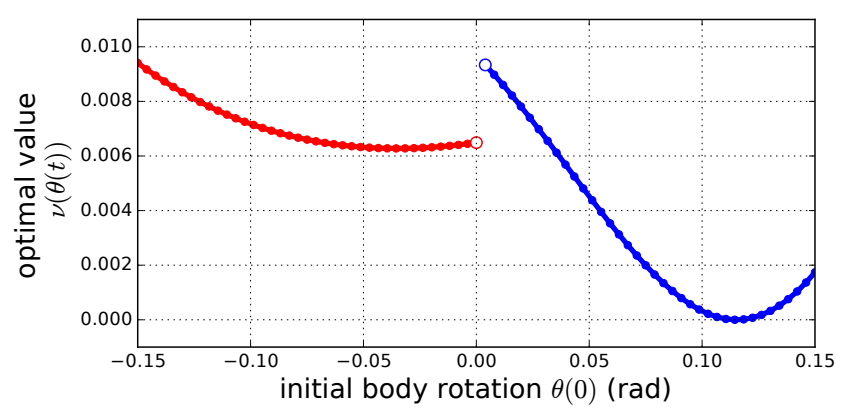

(f) optimal liftoff value

Fig. 4. Optimal trajectories, values and policies for touchdown and liftoff maneuvers. Optimizing (14, (15) for the biped in Fig. 11 yields trajectory outcomes $(\mathrm{a}, \mathrm{b})$, policies (c,d), and values (e,f) that are nonsmooth - piecewise-differentiable (left) or discontinuous (right). Asymmetries in trajectory outcomes are due to unequal input penalty parameters $\left(\alpha_{1} \neq \alpha_{2}\right)$ in (a) and unequal leg forces $\left(u_{1} \neq u_{2}\right)$ in $(\mathrm{b})$.

scalable algorithms, but does not ensure that policies optimized for the smoothed dynamics are (near-)optimal when applied to the original system's nonsmooth dynamics, since the dynamics of the smooth system being optimized differ from those of the original system. As an alternative approach, the framework we introduced in [15] provides design conditions that ensure trajectories of (1) depend continuouslydifferentiably on initial conditions. Thus in future work it may be possible to justify applying state-of-the-art algorithms for optimal control directly on some mechanical systems subject to unilateral constraints.

\section{B. Prevalence of nonsmoothness in contact-rich dynamics}

In Sec. IV we presented two optimal control problems where the dynamics of a mechanical system subject to unilateral constraints gave rise to a nonsmooth cost: one where the cost was piecewise-differentiable, and another where it was discontinuous. The reader may have noticed that the nonsmoothness occurred along trajectories that underwent simultaneous constraint (de)activation. This peculiarity was not accidental: in the absence of dry friction, the cost is generally continuously-differentiable along trajectories that (de)activate constraints at distinct time instants [31].

If the constraint surfaces intersect transversely [32, Ch. 6], then the nonsmoothness presented in Sec. IV is confined to a subset of the state space with zero Lebesgue measure. In light of this observation, intuition may lead one to ignore these states in practice. However, we believe this intuition will lead the practitioner astray as the complexity of considered behaviors increases. Indeed, since the number of contact mode sequences increases factorially with the number of constraints and exponentially with the number of constraint (de)activations, then the region where the cost function is continuously-differentiable is "carved up" into a 
rapidly increasing number of disjoint "pieces" as behavioral complexity increases.

Although we cannot at present comment in general on how these smooth pieces fit together, we note that some important behaviors will reside near a large number of pieces. For instance, periodic behaviors with (near-)simultaneous (de)activation of $n \in \mathbb{N}$ constraints as in [33] could yield up to $(n !)^{k}$ pieces after $k \in \mathbb{N}$ periods [34, Ch. 6]. The combinatorics are similar for tasks that involve intermittently activating (a subset of) $n$ constraints $k$ times as in [7]. Since the dimension of the state space is independent of $n$ and $k$, these pieces must be increasingly tightly packed as $n$ and/or $k$ increase.

Beyond the nonsmoothness induced by simultaneous constraint (de)activation considered here, mechanical systems subject to unilateral constraints can exhibit discontinuous or piecewise-differential trajectory outcomes due to dry friction, grazing, and bifurcations [35]-[37]. Such phenomena have been studied extensively from the perspective of nonsmooth dynamics and mechanics, but the implications for control have received comparatively little attention. It is our hope that the results presented herein will stimulate interest in this important application domain within the control community.

\section{REFERENCES}

[1] P. Ballard, "The dynamics of discrete mechanical systems with perfect unilateral constraints," Archive for Rational Mechanics and Analysis, vol. 154, no. 3, pp. 199-274, 2000.

[2] A. M. Johnson, S. A. Burden, and D. E. Koditschek, "A hybrid systems model for simple manipulation and self-manipulation systems," International Journal of Robotics Research, vol. 35, no. 11, pp. 1354 1392, 2016.

[3] E. Polak, Optimization: Algorithms and Consistent Approximations. Springer-Verlag, 1997.

[4] D. P. Bertsekas and J. N. Tsitsiklis, "Neuro-dynamic programming," Athena Scientific, 1996.

[5] M. Posa, C. Cantu, and R. Tedrake, "A direct method for trajectory optimization of rigid bodies through contact," International Journal of Robotics Research, vol. 33, no. 1, pp. 69-81, 2014.

[6] A. Patel, S. L. Shield, S. Kazi, A. M. Johnson, and L. T. Biegler, "Contact-Implicit trajectory optimization using orthogonal collocation," IEEE Robotics and Automation Letters, vol. 4, no. 2, pp. 22422249, 2019.

[7] I. Mordatch, E. Todorov, and Z. Popović, "Discovery of complex behaviors through contact-invariant optimization," ACM Transactions on Graphics, vol. 31, no. 4, pp. 43:1-43:8, 2012.

[8] A. Hereid, C. M. Hubicki, E. A. Cousineau, and A. D. Ames, "Dynamic humanoid locomotion: A scalable formulation for HZD gait optimization," IEEE Transactions on Robotics, vol. 34, no. 2, pp. 370$387,2018$.

[9] J. Schulman, S. Levine, P. Moritz, M. I. Jordan, and P. Abbeel, "Trust region policy optimization," CoRR, abs/1502. 05477, 2015.

[10] V. Kumar, E. Todorov, and S. Levine, "Optimal control with learned local models: Application to dexterous manipulation," in IEEE International Conference on Robotics and Automation, 2016, pp. 378-383.

[11] S. Levine, C. Finn, T. Darrell, and P. Abbeel, "End-to-end training of deep visuomotor policies," Journal of Machine Learning Research: JMLR, vol. 17, no. 1, pp. 1334-1373, 2016.

[12] J. Hwangbo, J. Lee, A. Dosovitskiy, D. Bellicoso, V. Tsounis, V. Koltun, and M. Hutter, "Learning agile and dynamic motor skills for legged robots," Science Robotics, vol. 4, no. 26, p. eaau5872, 2019.

[13] R. J. Williams, "Simple statistical gradient-following algorithms for connectionist reinforcement learning," Machine Learning, vol. 8, no. 3-4, pp. 229-256, 1992.

[14] A. M. Pace and S. A. Burden, "Piecewise-differentiable trajectory outcomes in mechanical systems subject to unilateral constraints," in Hybrid Systems: Computation and Control, 2017.
[15] —, "Decoupled limbs yield differentiable trajectory outcomes through intermittent contact in locomotion and manipulation," in IEEE International Conference on Robotics and Automation, 2017.

[16] C. D. Remy, K. Buffinton, and R. Siegwart, "Stability analysis of passive dynamic walking of quadrupeds," International Journal of Robotics Research, vol. 29, no. 9, pp. 1173-1185, 2010.

[17] Y. Hürmüzlü and D. B. Marghitu, "Rigid body collisions of planar kinematic chains with multiple contact points," International Journal of Robotics Research, vol. 13, no. 1, pp. 82-92, 1994.

[18] S. Scholtes, Introduction to Piecewise Differentiable Equations. Springer-Verlag, 2012.

[19] R. W. Chaney, "Second-Order sufficient conditions in nonsmooth optimization," Mathematics of Operations Research, vol. 13, no. 4, pp. 660-673, 1988.

[20] S. M. Robinson, "An Implicit-Function theorem for a class of nonsmooth functions," Mathematics of Operations Research, vol. 16, no. 2 , pp. 292-309, 1991.

[21] S. Burden, H. Gonzalez, R. Vasudevan, R. Bajcsy, and S. Sastry, "Metrization and Simulation of Hybrid Control Systems," IEEE Transactions on Automatic Control, vol. 60, no. 9, pp. 2307-2320, 2015.

[22] R. S. Sutton, D. McAllester, S. Singh, and Y. Mansour, "Policy gradient methods for reinforcement learning with function approximation," Neural Information Processing Systems, vol. 12, pp. 1057-1063, 2000.

[23] J. Baxter and P. Bartlett, "Infinite-horizon policy-gradient estimation," Journal of Artificial Intelligence Research, vol. 15, pp. 319-350, 2001.

[24] S. Kakade, "A natural policy gradient," Neural Information Processing Systems, vol. 14, pp. 1531-1538, 2001.

[25] K. Doya, "Reinforcement learning in continuous time and space," Neural Computation, vol. 12, no. 1, pp. 219-245, 2000.

[26] V. R. Konda and J. N. Tsitsiklis, "On Actor-Critic algorithms," SIAM Journal on Control and Optimization, vol. 42, no. 4, pp. 1143-1166, 2003.

[27] D. Silver, G. Lever, N. Heess, T. Degris, D. Wierstra, and M. Riedmiller, "Deterministic Policy Gradient Algorithms," in ICML, Beijing, China, 2014.

[28] S. Levine and P. Abbeel, "Learning neural network policies with guided policy search under unknown dynamics," in Neural Information Processing Systems 27, 2014, pp. 1071-1079.

[29] T. Erez and E. Todorov, "Trajectory optimization for domains with contacts using inverse dynamics," in IEEE International Conference on Intelligent Robots and Systems, 2012, pp. 4914-4919.

[30] I. Mordatch, K. Lowrey, and E. Todorov, "Ensemble-CIO: Full-body dynamic motion planning that transfers to physical humanoids," in IEEE International Conference on Intelligent Robots and Systems, 2015, pp. 5307-5314.

[31] M. A. Aizerman and F. R. Gantmacher, "Determination of stability by linear approximation of a periodic solution of a system of differential equations with discontinuous Right-Hand sides," Quarterly Journal of Mechanics and Applied Mathematics, vol. 11, no. 4, pp. 385-398, 1958.

[32] J. M. Lee, Introduction to Smooth Manifolds, 2nd ed., ser. Graduate texts in mathematics. New York ; London: Springer, 2012.

[33] R. M. Alexander, "Gaits of bipedal and quadrupedal animals," International Journal of Robotics Research, vol. 3, no. 2, pp. 49-59, 1984.

[34] B. S. Banjanin, "Data-driven modeling for hybrid dynamical systems," Ph.D. dissertation, University of Washington, 2019.

[35] A. B. Nordmark, "Universal limit mapping in grazing bifurcations," Physical Review E, vol. 55, no. 1, pp. 266-270, 1997.

[36] M. d. Bernardo, C. J. Budd, A. R. Champneys, P. Kowalczyk, A. B. Nordmark, G. O. Tost, and P. T. Piiroinen, "Bifurcations in nonsmooth dynamical systems," SIAM Review, vol. 50, no. 4, pp. 629-701, 2008.

[37] O. Makarenkov and J. S. W. Lamb, "Dynamics and bifurcations of nonsmooth systems: A survey," Physica D. Nonlinear phenomena, vol. 241 , no. 22 , pp. 1826-1844, 2012. 